\title{
Review on Bile Acids: Effects of the Gut Microbiome, Interactions with Dietary Fiber, and Alterations in the Bioaccessibility of Bioactive Compounds
}

Jashbir Singh, Rita Metrani, Siddanagouda R. Shivanagoudra,

Guddadarangavvanahally K. Jayaprakasha, * and Bhimanagouda S. Patil*

Vegetable and Fruit Improvement Center, Department of Horticultural Sciences, Texas A\&M

University, 1500 Research Parkway, Suite A120, College Station, Texas 77845, United States 

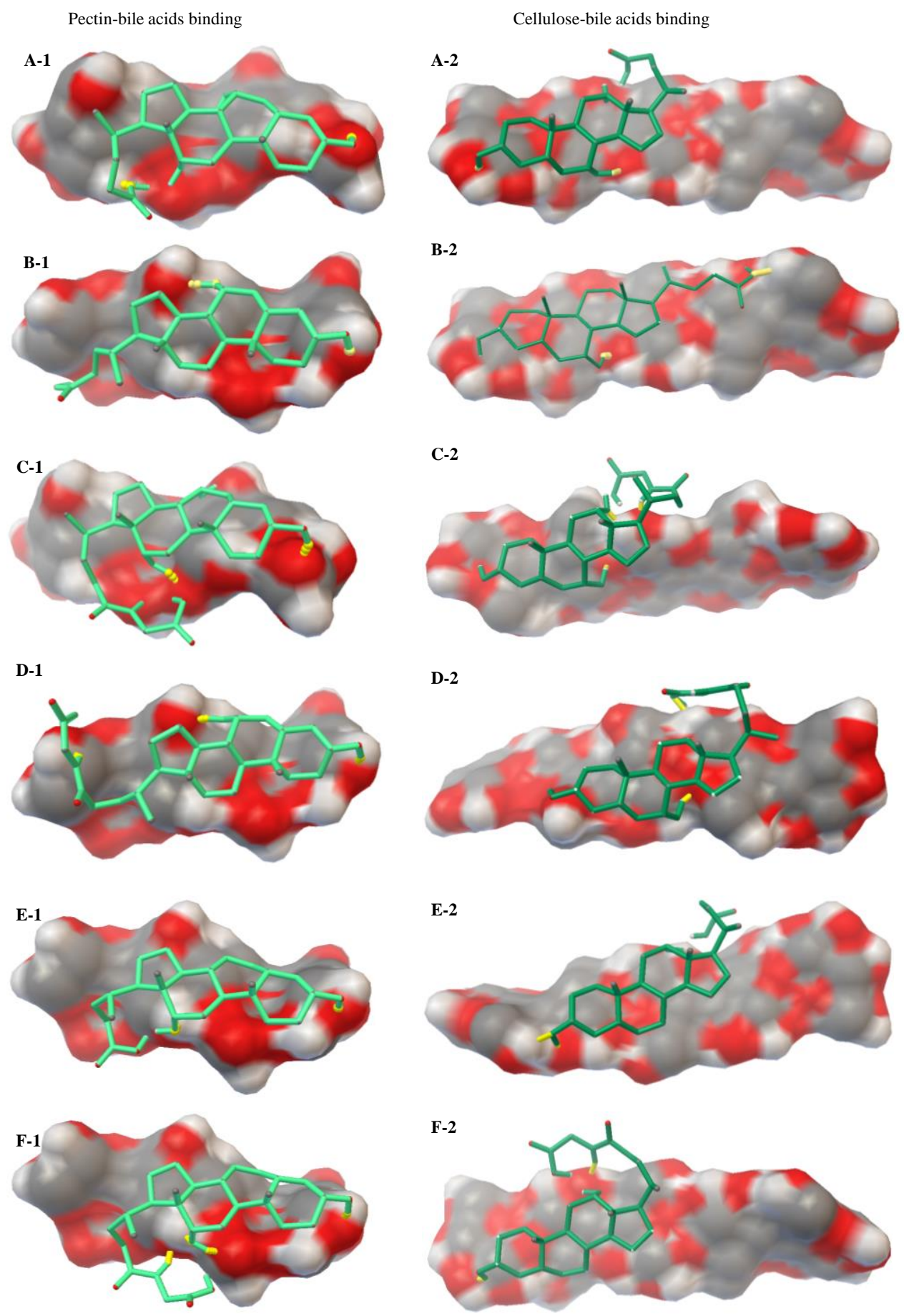

Figure S1. 3D hypothetical molecular docking representation of the interaction between pectin (A-1 to F1) and cellulose (A-2 to F-2) with different bile acid salts. Bile acids are shown in stick representation and pectin and cellulose are represented as a molecular surface. Hydrogen bonds are indicated as yellow lines. The binding sites of bile salts and pectin/cellulose are represented as a red-white transparent hull. The letters refer to: A, cholate; B chenodeoxycholate; C, glycocholate; D, glycochenodeoxycholate; E, deoxycholate; F, glycodeoxycholate 